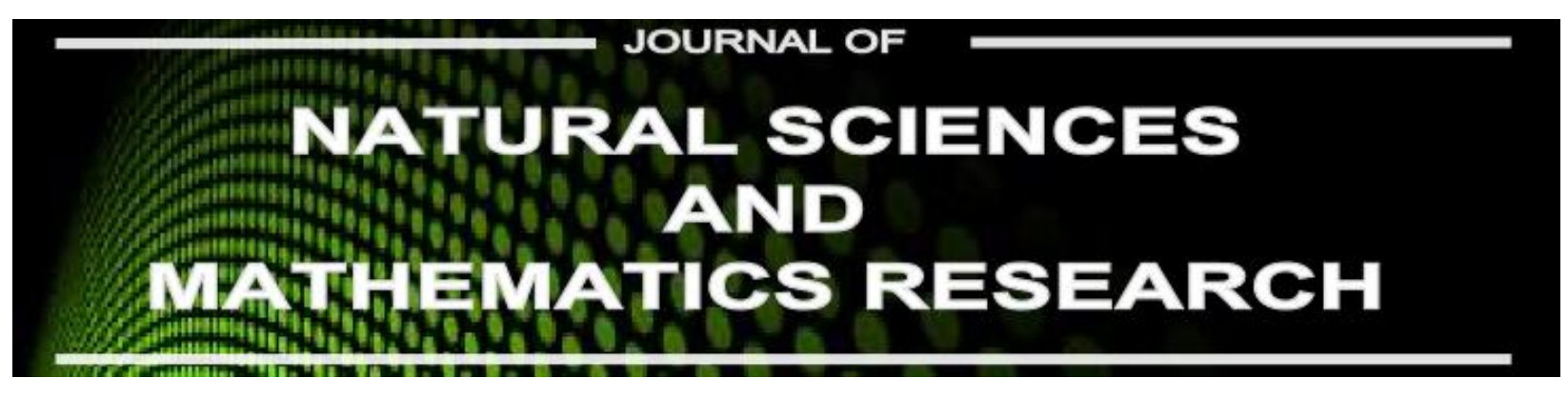

Available online at http://journal.walisongo.ac.id/index.php/jnsmr

\title{
Desaign of Converter Hijri Calendar to Ad Calendar for Determaning Islamic Great Days Using Matlab
}

\section{Uzlifa Khanifatul Muttaqi}

Department of Mathematics Education, Universitas Islam Negeri Walisongo Semarang, Central Java, Indonesia

\begin{abstract}
s
Corresponding author: zlyfa_khanyfa@yahoo.co.id Recived: 25 March 2016, Revised: 02 April 2016 Accepted: 15 June 2016.

This research is motivated by the importance of knowing an Islamic great day calendar on $\mathrm{AD}$ calendar. It was intended to answer the question: How to design converter Hijri calendar to AD calendar for determining Islamic great days using Matlab? The research used 'design' method, because it was one of activities to translate the result of analysis to software form and also used to develop an existing application. This application made by a Matlab software. Matlab was a conversion tool to determine Islamic great days. Data of this research was obtained from questionnaire. And all the questionnaire datum was used to design application. The technique of feasibility analysis application was obtained from a validation. It was using black-box method. The result showed that the application was made by three ways: First, making an appearance in GUI Matlab appropriate with interface design application. Second, entering the script in each menu of application. Third, entering JD formula in application that were already functioning. (C2016 JNSMR UIN Walisongo. All rights reserved.
\end{abstract}

Key words: Matlab; Converter; AD Calendar; Hijri Calendar.

\section{Introduction}

In this decade, the number theory became a development basis of several branches of mathematics study, such as cryptography (hieroglyph or password), calendar, and computer science [1]. This research used number theory concept to determine various dating systems [2,3]. The concept used divisibility and congruence. Human needed a date or calendar in life. As the definition of dating was an unit of time measurement used by human to record significant or historical events, even in human life or surrounding environment [4]. The unit of time measurement were days, weeks, months, years, and etc. For Moslem, calendar was an important requirement. Therefore, calendar system was also urgently needed, because time can be known currently and simply. Based on questionnaires result, it found that: 1) $100 \%$ of math students needed a calendar. $65 \%$ of them 
could plan their activities, $6 \%$ knew a date, and $5 \%$ knew days off, and $55 \%$ of them were not able to practice properly. 2) Most of math students did not know that mathematical number theory could be applied for calendar calculation. 3) From the various software they studied, most of them only used Matlab to create learning media. 4) Most of students never used the applications to determine Islamic great days. $15 \%$ of students used the application to find out the Islamic great days felt difficultly. Because, the apps poorly understood by user who did not understand astronomy science and the apps also was not simple.

In addition, calendar to determine moon seen (ru'yah) and moon calculation (hisab) debate [5], along with knowledge development and technological progress was a variety tools. According both of them or other methods, there were many applications to determine calendar system that had created, such as Accurate Hijri Calculator (application to specify Hijri-AD month and to determine prayer time in several countries), Stellium (application to determine BC year by knowing weather and moon location), and Accurate Time (application to determine new moon position and prayer time. This tools were created to facilitate human in determining a calendar. The calendar was created by a computer. The computer was controlled by set of instructions it was called by program. This research used the application which made by a computer program, known by Matlab [6,7], to load the calendar system.

\section{Desaign of Converter Hijri Calendar}

\section{Analysis of User Needs}

Calendar is important especially for math education students and generally for moslem. It also can be called that the calendar calculation is not only needs but also obligation, because by knowing calendar calculation, moslem be able to know: 1) the appointment time of obligatory worship, such as Ramadlan fasting, forbidden fasting time, hajj time, and forbidden war time. 2) the appointment time of optional (sunnah) and treatment (mubah) worship, such as recommendation of Muharram fasting at 9 and 10, compensation for orphan, Muhammad prophet birth anniversary or usually called by 'Maulid Nabi', isra' and mi'raj anniversary, and nuzulul qur'an anniversary[8].

\section{Design 'Converter Calendar' Application}

1. Implementation of 'Calendar Converter' Application Code

Implementation of the application code is made after a known algorithm application creation. The Figure 1, Figure 2, Figure 3, and Figure 4 are the implementation of the code of applications code that created with Flowchart.

2. Step Incorporate Apps 'Script'

a. Insert the 'script' on the button Conversion. Write the 'script' in the buttom 'Function Konversi_Callback', by input the following 'script':

InputTanggal $=$

str2double(get(handles.InputTanggal, 'string'));

InputBulan =

str2double(get(handles.InputBulan,'string'));

InputTahun =

str2double(get(handles.InputTahun,'string'));

TahunHijriah=InputTahun-1;

NomerTahunHijriah=fix(TahunHijriah/30);

SisaTahunHijriah= TahunHijriah-

(NomerTahunHijriah*30);

HariHijriah=NomerTahunHijriah*10631;

if SisaTahunHijriah $<2$

KabisatHijriah=0;

else if SisaTahunHijriah $<5$

KabisatHijriah=1;

else if SisaTahunHijriah $<7$

KabisatHijriah=2;

else if SisaTahunHijriah < 10

KabisatHijriah=3;

else if SisaTahunHijriah $<13$

KabisatHijriah=4;

else if SisaTahunHijriah $<16$

KabisatHijriah=5;

else if SisaTahunHijriah < 18

KabisatHijriah=6;

else if SisaTahunHijriah $<21$

KabisatHijriah=7;

else if SisaTahunHijriah $<24$

KabisatHijriah=8;

else if SisaTahunHijriah $<26$ 


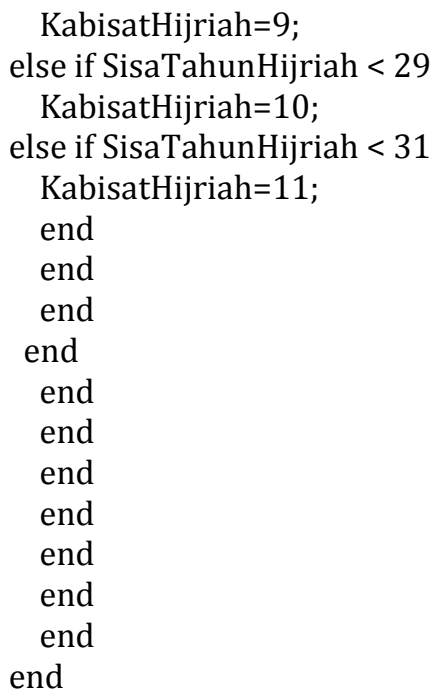

SisaHariHijriah=SisaTahunHijriah*354+KabisatH ijriah;

if InputBulan $==1$

JumlahInputBulan=0;

else if InputBulan $==2$

JumlahInputBulan=30;

else if InputBulan==3

JumlahInputBulan=59;

else if InputBulan==4

JumlahInputBulan=89;

else if InputBulan $==5$

JumlahInputBulan=118;

else if InputBulan $==6$

JumlahInputBulan=148;

else if InputBulan $==7$

JumlahInputBulan=177;

else if InputBulan $==8$

JumlahInputBulan=207;

else if InputBulan $==9$

JumlahInputBulan=236;

else if InputBulan==10

JumlahInputBulan=266;

else if InputBulan==11

JumlahInputBulan=295;

else if InputBulan==12

JumlahInputBulan=325;

end

end

end

end

end

end

end

end

end

JumlahHijriah=HariHijriah + SisaHariHijriah + JumlahInputBulan + InputTanggal;
JD=1948438.5+JumlahHijriah;

JD1=JD+0.5;

$\mathrm{Z}=\mathrm{fix}(J \mathrm{D} 1)$;

$\mathrm{F}=\mathrm{JD} 1-\mathrm{Z}$;

$\mathrm{AA}=\mathrm{fix}((\mathrm{Z}-1867216.25) / 36524.25)$;

$\mathrm{A}=\mathrm{Z}+1+\mathrm{AA}-\mathrm{fix}(\mathrm{AA} / 4)$;

if $\mathrm{Z}<2299161$

$\mathrm{A}=\mathrm{Z}$;

end

$\mathrm{B}=\mathrm{A}+1524$;

$\mathrm{C}=\mathrm{fix}((\mathrm{B}-122.1) / 365.25)$;

$\mathrm{D}=\mathrm{fix}\left(365.25^{*} \mathrm{C}\right)$;

$E=f i x((B-D) / 30.6001)$;

HasilTanggal=fix(B-D-fix $(30.6001 * E)+F)$;

set (handles.HasilTanggal,'string',

num2str(HasilTanggal));

HasilBulan=E-1;

if $\mathrm{E}==14$

HasilBulan=E-13;

else if $E==15$

HasilBulan=E-13;

end

end

set (handles.HasilBulan, 'string',num2str

(HasilBulan));

HasilTahun=C-4716;

if HasilBulan $==1$

HasilTahun=C-4715;

else if HasilBulan==2

HasilTahun=C-4715;

end

end

set(handles.HasilTahun,'string',num2str(HasilTa

hun));

NomerHariMasehi=JD+1.5;

HasilHari= fix $(\bmod ($ NomerHariMasehi,7) +1$)$;

if HasilHari==1

HasilHari =sprintf('Minggu');

set (handles.HasilHari, 'string',HasilHari);

elseif HasilHari $==2$

HasilHari =sprintf('Senin');

set (handles.HasilHari, 'string',HasilHari);

elseif HasilHari $==3$

HasilHari =sprintf('Selasa');

set (handles.HasilHari, 'string',HasilHari);

elseif HasilHari $==4$

HasilHari =sprintf('Rabu');

set (handles.HasilHari, 'string',HasilHari);

elseif HasilHari $==5$

HasilHari =sprintf('Kamis');

set (handles.HasilHari, 'string',HasilHari);

elseif HasilHari $==6$

HasilHari =sprintf('Jumat');

set (handles.HasilHari, 'string',HasilHari); 
elseif HasilHari==7

HasilHari =sprintf('Sabtu');

set (handles.HasilHari, 'string',HasilHari);

end

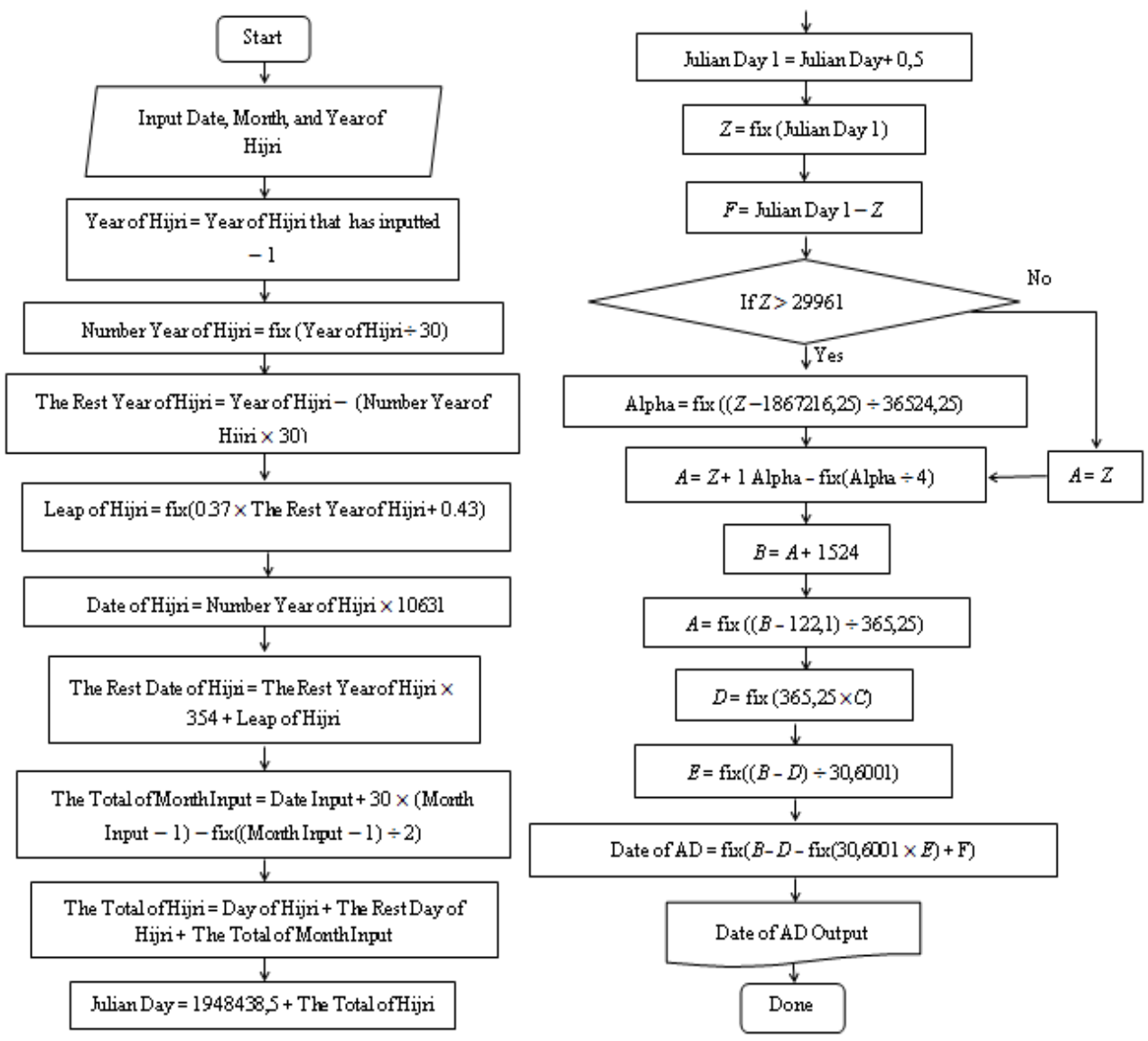

Figure 1. Date of AD Searching Flowchart

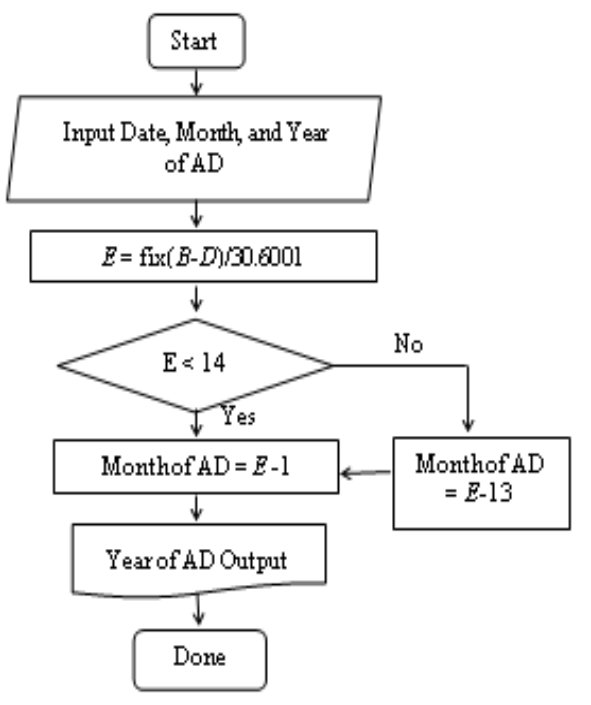

Figure 2. Month of AD Searching Flowchart

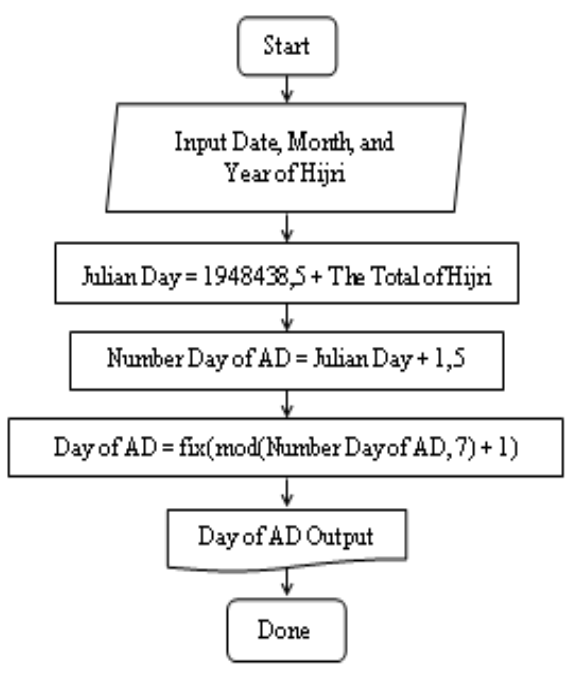

Figure 3. Conversion Hijri to AD Searching Flowchart 


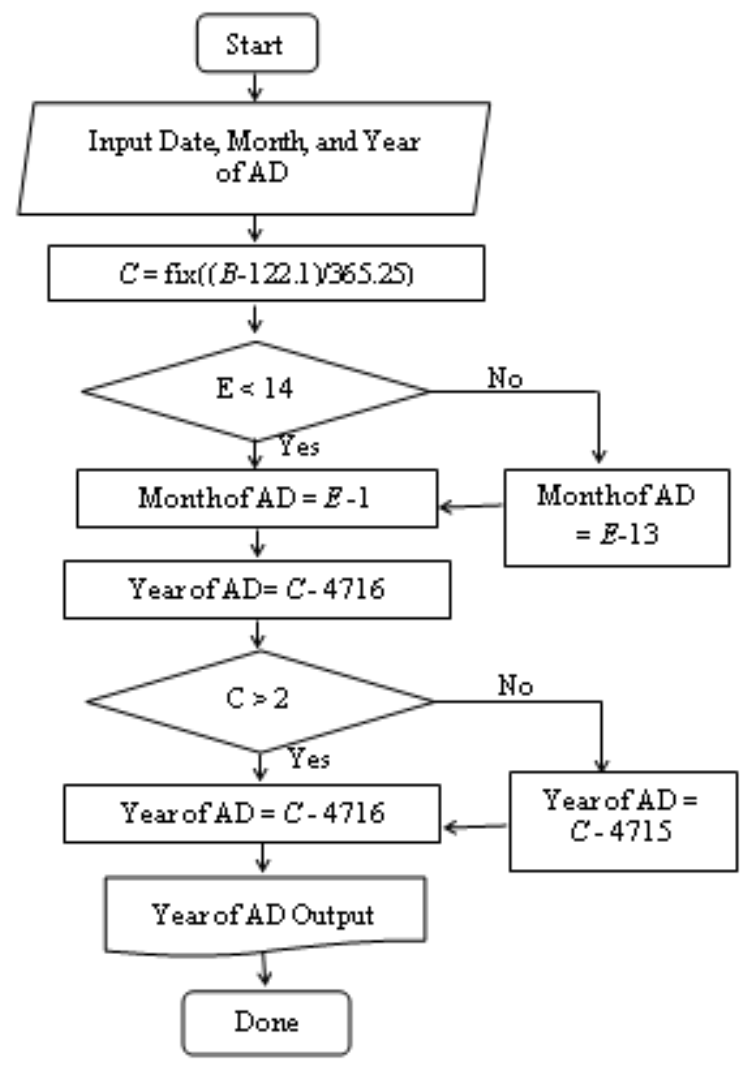

Figure 4. Year of AD Searching Flowchart

b. Insert the 'script' on the Refresh button. Write the 'script' in the buttom of 'Function Refresh_Callback', with the 'script' as follows:

set (handles.InputTannggal, 'string', "); set (handles.InputBulan, 'string', "); set (handles.InputTahun, 'string', "); set (handles.HasilHari, 'string', "); set (handles.HasilTanggal, 'string', "); set (handles.HasilBulan, 'string', "); set (handles.HasilTahun, 'string', ");

c. Insert the 'script' on the Close button. Write the 'script' in the buttom 'Function Refresh_Callback', with the 'script' as follows:

$$
\text { close; }
$$

d. Insert the 'script' on the '?' button. Write the 'script' under 'Function Catatan_Callback', with the 'script' as follows:

response $=$ Notes;

if Close close (handles, response);

The Results of 'Calendar Converter' Application

The Figure 5 is the final results of 'Calendar Converter' applications.

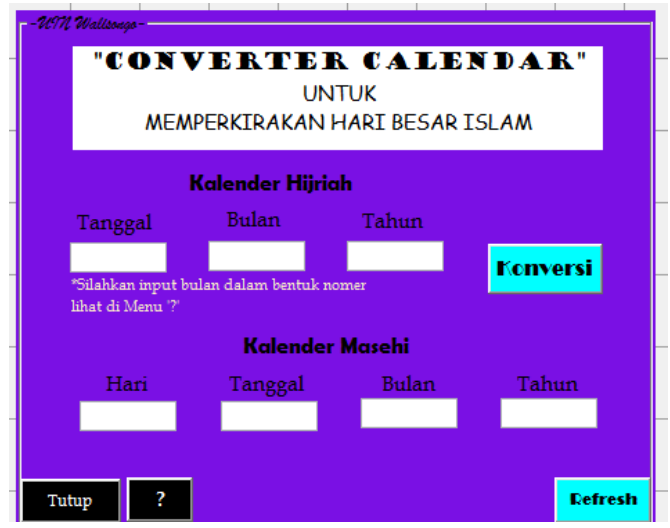

Figure 5. The Final Results Display of 'Calendar Converter' Applications 


\section{Testing 'Converter Calendar' Applications}

The test results obtained from the two stages, those are 1) Test results conducted by comparing between the applications that made to the schedule of Islamic great days and that decided by the Ministry of Religion, namely Muharram, Ramadan, Eid al-Fitr and Eid al-
Adha, the results shown in the Table 1, 2) Test result conducted comparison of the predicted 5 years, starting in $1442 \mathrm{H}$, the results shown in the Table 2.

Table 1. The Test Result Applications o Islamic Great Days and Ministry Religion Schedule since 1427 to 1431 Hijri

\begin{tabular}{|c|c|c|c|c|}
\hline No. & Year & $\begin{array}{l}\text { Great Day of } \\
\text { Islam }\end{array}$ & $\begin{array}{l}\text { According to Ministry of } \\
\text { Religion Calendar }\end{array}$ & Date Converter \\
\hline \multirow{4}{*}{1.} & \multirow{4}{*}{1427} & 1 Muharram & Tuesday, 31 of January 2006 & Suitable \\
\hline & & 1 Ramadlan & Monday, 24 of September 2006 & Suitable \\
\hline & & I Syawal & Tuesday, 24 October 2006 & Suitable \\
\hline & & 10 Dzulhijjah & Monday, 31 December 2006 & Suitable \\
\hline \multirow{4}{*}{2.} & \multirow{4}{*}{1428} & 1 Muharram & Saturday, 21 January 2007 & Saturday, 20 January 2007 \\
\hline & & 1 Ramadlan & Thursday, 13 September 2007 & Suitable \\
\hline & & I Syawal & Saturday, 13 October 2007 & Suitable \\
\hline & & 10 Dzulhijjah & Thursday, 20 December 2007 & Suitable \\
\hline \multirow{4}{*}{3.} & \multirow{4}{*}{1429} & 1 Muharram & Wednesday, 10 January 2008 & Suitable \\
\hline & & 1 Ramadlan & Monday, 1 September 2008 & Tuesday, 2 September 2008 \\
\hline & & I Syawal & Wednesday, 1 October 2008 & Suitable \\
\hline & & 10 Dzulhijjah & Monday, 8 December 2008 & Tuesday, 9 December 2008 \\
\hline \multirow{4}{*}{4.} & \multirow{4}{*}{1430} & 1 Muharram & Monday, 29 December 2008 & Suitable \\
\hline & & 1 Ramadlan & Friday, 22 August 2009 & Suitable \\
\hline & & I Syawal & Sunday, 20 September 2009 & Monday, 21 September 2009 \\
\hline & & 10 Dzulhijjah & Friday, 27 November 2009 & Saturday, 28 November 2009 \\
\hline \multirow{4}{*}{5.} & \multirow{4}{*}{1431} & 1 Muharram & Friday, 18 December 2009 & Suitable \\
\hline & & 1 Ramadlan & Thursday, 11 August 2010 & Suitable \\
\hline & & I Syawal & Friday, 10 September 2010 & Suitable \\
\hline & & 10 Dzulhijjah & Wednesday, 17 November 2010 & Suitable \\
\hline
\end{tabular}


Table 2. The Test Result Conducted Comparison of the Predicted 5 Years Later

\begin{tabular}{|c|c|c|c|c|c|}
\hline No. & Year & $\begin{array}{c}\text { The Number of } \\
\text { Great Day of } \\
\text { Islam }\end{array}$ & NASA Calendar & $\begin{array}{l}\text { Predicted of Ministry } \\
\text { Religion Calendar }\end{array}$ & $\begin{array}{c}\text { Date } \\
\text { Converter }\end{array}$ \\
\hline \multirow{4}{*}{1.} & \multirow{4}{*}{1442} & 1 Muharram & $\begin{array}{c}\text { Wednesday, } 19 \text { August } \\
2020\end{array}$ & $\begin{array}{l}\text { Wednesday, } 20 \text { August } \\
2020\end{array}$ & Suitable \\
\hline & & 1 Ramadlan & Monday, 12 April 2021 & Tuesday, 13 April 2021 & Suitable \\
\hline & & I Syawal & $\begin{array}{c}\text { Wednesday, } 12 \text { May } \\
2021\end{array}$ & Thursday, 13 May 2021 & Suitable \\
\hline & & 1 Dzulhijjah & Saturday, 10 July 2021 & Sunday, 11 July 2021 & Suitable \\
\hline \multirow{4}{*}{2.} & \multirow{4}{*}{1443} & 1 Muharram & Sunday, 8 August 2021 & Tuesday, 10 August 2021 & $\begin{array}{c}\text { Suitable } \\
\text { Sunday, } 3\end{array}$ \\
\hline & & 1 Ramadlan & Friday, 1 April 2022 & Saturday, 2 April 2022 & $\begin{array}{l}\text { April } \\
2022\end{array}$ \\
\hline & & I Syawal & Sunday, 1 May 2022 & Monday, 2 May 2022 & $\begin{array}{c}\text { Tuesday, } \\
3 \text { May } \\
2022\end{array}$ \\
\hline & & 1 Dzulhijjah & $\begin{array}{c}\text { Wednesday, } 29 \text { June } \\
2022\end{array}$ & Friday, 1 July 2022 & Suitable \\
\hline \multirow{3}{*}{3.} & \multirow{3}{*}{1444} & 1 Muharram & Friday, 29 July 2022 & Saturday, 30 July 2022 & Suitable \\
\hline & & 1 Ramadlan & $\begin{array}{c}\text { Wednesday, } 22 \text { March } \\
2023\end{array}$ & Thursday, 23 March 2023 & Suitable \\
\hline & & $\begin{array}{c}\text { I Syawal } \\
1 \text { Dzulhijjah }\end{array}$ & $\begin{array}{c}\text { Thursday, } 20 \text { April } 2023 \\
\text { Sunday, } 18 \text { June } 2023\end{array}$ & $\begin{array}{l}\text { Saturday, } 22 \text { April } 2023 \\
\text { Tuesday, } 20 \text { June } 2023\end{array}$ & $\begin{array}{l}\text { Suitable } \\
\text { Suitable }\end{array}$ \\
\hline \multirow{4}{*}{4.} & \multirow{4}{*}{1445} & 1 Muharram & Tuesday, 18 July 2023 & Wednesday, 19 July 2023 & $\begin{array}{l}\text { Suitable } \\
\text { Monday, }\end{array}$ \\
\hline & & 1 Ramadlan & Sunday, 10 March 2024 & Tuesday, 12 March 2024 & $\begin{array}{l}11 \text { March } \\
2024\end{array}$ \\
\hline & & I Syawal & Tuesday, 9 April 2024 & Wednesday, 10 April 2024 & Suitable \\
\hline & & 1 Dzulhijjah & Friday, 7 June 2024 & Saturday, 8 June 2024 & Suitable \\
\hline \multirow{4}{*}{5.} & \multirow{4}{*}{1446} & 1 Muharram & Thursday, 4 July 2024 & Sunday, 7 July 2024 & $\begin{array}{l}\text { Monday, } 8 \\
\text { July } 2024\end{array}$ \\
\hline & & 1 Ramadlan & $\begin{array}{c}\text { Friday, } 28 \text { February } \\
2025\end{array}$ & Saturday, 1 March 2025 & Suitable \\
\hline & & I Syawal & $\begin{array}{c}\text { Saturday, } 29 \text { March } \\
2025\end{array}$ & Monday, 31 March 2025 & Suitable \\
\hline & & 1 Dzulhijjah & Tuesday, 27 May 2025 & Thursday, 29 May 2025 & Suitable \\
\hline
\end{tabular}

Validation 'Calendar Converter' Application

The Table 3 is the test case to validate the application using black-box. 
Table 3. Black-box Validation Result Application

\begin{tabular}{|c|c|c|c|c|}
\hline No. & Application Unit & Design Process & The Expected Result & Notes \\
\hline \multirow{3}{*}{1.} & \multirow{3}{*}{$\begin{array}{l}\text { Data of Input Menu } \\
\text { Hijri Calendar }\end{array}$} & Click Date Menu & Date Selection Number Display & Accordance \\
\hline & & Click Month Menu & Month Selection Number Display & Accordance \\
\hline & & Write Year Menu Wanted & Year Selection Number Display & Accordance \\
\hline 2. & Process Menu & Click Conversion Button & $\begin{array}{c}\text { Data Result of AD Calendar } \\
\text { Display }\end{array}$ & Accordance \\
\hline \multirow{4}{*}{3.} & \multirow{4}{*}{$\begin{array}{c}\text { AD Calendar Result } \\
\text { Menu }\end{array}$} & Click Conversion Button & Day Result Display & Accordance \\
\hline & & Click Conversion Button & Date Result Display & Accordance \\
\hline & & Click Conversion Button & Month Result Display & Accordance \\
\hline & & Click Conversion Button & Year Result Display & Accordance \\
\hline 4. & Delete Menu & Click Delete Button & $\begin{array}{c}\text { Deleting Year Data of Hijri } \\
\text { Calendar and data results of AD } \\
\text { Calendar }\end{array}$ & Accordance \\
\hline 5. & Close Menu Apps & Click Close Button & The Application is Closed & Accordance \\
\hline 6. & Menu '?' & Click '?'Button & $\begin{array}{l}\text { List of Hijri and AD Calendar } \\
\text { Number Display }\end{array}$ & Accordance \\
\hline
\end{tabular}

\section{Conclusion}

This application was made by: First, making an appearance in GUI Matlab appropriate with the application interface design. Second, entering the script on each menu of application. Third, entering the JD formula on applications that were already functioning. Then, the application was ready to use by this way: input date, month, and year of Hijri calendar in input menu. And the output result of it was day, date, month, and year of AD calendar.

\section{Acknowledgment}

The author wish to thank Department of Mathematics Education, Faculty of Sciences and Technology, Unversitas Islam Negeri Walisongo Semarang for financial support in this research.

\section{References}

[1] R. Anugraha, Mekanika Benda Langit. Yogyakarta: Lab. Fisika Material dan
Instrumentasi Jurusan Fisika FMIPA UGM. 2012.

[2] A.H. Fathani, Hakikat Matematika dan Logika. Jogjakarta: Ar-Ruzz Media. 2009.

[3] E. Utami, et al. 10 Langkah Belajar Logika dan Algoritma Menggunakan Bahasa C dan C++ di GNU/LINUX. Yogyakarta: Andi Offset,2005.

[4] S. Azhari, Ensiklopedi Hisab Ru'yah. Yogyakarta: Pustaka Pelajar. Type-2. 2008.

[5] . Menimbang Kalender Islam dan Masehi. http://rinto.staff.ugm.ac.id. access at $14^{\text {th }}$ of February 2016.

[6] M. Arhami, Pemograman MATLAB. Yogyakarta: Andi Offset, 2005.

[7] G.A. Away, The Shortcut of MATLAB Programming. Bandung: Informatika. 2014.

[8] Depertemen Agama RI. Al-Qur'an dan Terjemahnya. Bekasi: Cipta Bagus Segara. 2012. 